\title{
MÖSSBAUER EVIDENCE FOR A SPIN-COMPENSATED STATE IN DILUTE Fe-Cu ALLOYS
}

R. B. Frankel, N. A. Blum,* Brian B. Schwartz, and Duk Joo Kim National Magnet Laboratory, $\dagger$ Massachusetts Institute of Technology, Cambridge, Massachusetts (Received 10 May 1967)

There has been considerable recent interest in the existence and nature of the bound state $^{1-9}$ which is thought to be formed in certain dilute alloys between localized magnetic impurity moments and conduction-electron spins below some critical temperature characteristic of the alloy. In their recent communication, Daybell and Steyert ${ }^{10}$ presented evidence based on low-temperature resistivity and susceptibility measurements for the formation of such a bound state consistent with some quenching of the localized moment associated with very dilute $\mathrm{Fe}$ in $\mathrm{Cu}$. The results of Mössbauer experiments on dilute $\mathrm{Fe}$ in $\mathrm{Cu}$ in high external magnetic fields (42-136 kOe) are presented here as evidence for significant destruction of the bound state by magnetic fields for which $\mu H_{0} \sim k T_{\mathrm{K}}$ where $k T_{\mathrm{K}}$ is on the order of the energy change associated with the formation of the bound state. The fact that the Mössbauer hyperfine spectrum reflects the electronic environment within atomic dimensions of the ${ }^{57} \mathrm{Fe}$ nucleus enables us to make some conjectures as to the singlet nature of the bound state.

The hyperfine interaction in dilute $\mathrm{Fe}$ in $\mathrm{Cu}$ alloys has been reported by Kitchens, Steyert, and Taylor ${ }^{11}$ using the Morssbauer technique in external magnetic fields up to $62 \mathrm{kOe}$ and at temperatures down to $0.4^{\circ} \mathrm{K}$. They noted significant deviations from pure paramagnetic behavior and interpreted their results in terms of a model due to Housley and Dash, ${ }^{12}$ who introduced a phenomenological interaction between the localized Fe spin and conduction-electron spindensity waves. Measurements at higher fields $\left(H_{0}=110 \mathrm{kOe}\right)$ were reported by Blum, Freeman, and Grodzins. ${ }^{13}$ In the ideal paramagnetic case the hyperfine field $H_{\mathrm{hf}}$ is proportional to a Brillouin function characterized by the

$$
\begin{aligned}
& \text { parameters } J, g \text {, and } H_{\text {sat }}{ }^{14} \text { : } \\
& \qquad H_{\mathrm{hf}}=H_{\text {sat }}{ } J\left(g \mu_{\mathrm{B}} H_{0} / k T\right),
\end{aligned}
$$

where $J$ is the total angular momentum associated with the paramagnetic moment, $g$ is the $g$ factor, and $H_{\text {sat }}$ is the magnitude of the hyperfine field for sufficiently large values of $H_{0} / T$. The sign of $H_{\text {hf }}$ for $\mathrm{Fe}$ in $\mathrm{Cu}$ is negative, i.e., opposite to the direction of the magnetic moment. In the simplest cases the value of $H_{\text {sat }}$ has been shown to be proportional to the magnitude of the moment localized on the impurity site and does not change as a function of applied magnetic field, neglecting small Knight-shift contributions. ${ }^{14}$ In the Fe-Cu system, however, for the fields and lowest temperatures used $\left(42 \leqslant H_{0} \leqslant 136 \mathrm{kOe} ; T \simeq 1.1^{\circ} \mathrm{K}\right)$, we find that although the effective localized moments are fully polarized in the sense that decreasing $T$ does not change the value of the observed hyperfine field, the magnitude of the saturation hyperfine field $\left|H_{\text {sat }}\right|$ does depend upon the magnitude of the externally applied field, and, as shown in Figs. 1 and 2, increases monotonically as $H_{0}$ increases. The experiments were performed using several sources of ${ }^{57} \mathrm{Co}$ plated onto pure $(99.999 \%)$ copper foil, annealed in hydrogen at $850^{\circ} \mathrm{C}$ for several hours and quenched to room temperature. The experimental results are essentially independent of the source used. Source strengths varied from 10 to $100 \mathrm{mCi}$ and the impurity concentration $(\mathrm{Fe}+\mathrm{Co})$ is estimated from 100 to $1000 \mathrm{ppm}$ for the various sources.

In view of the recent resistivity and susceptibility results, ${ }^{10}$ our Mössbauer data may be explained on the basis of a spin-compensated state appearing at low temperatures and fields 


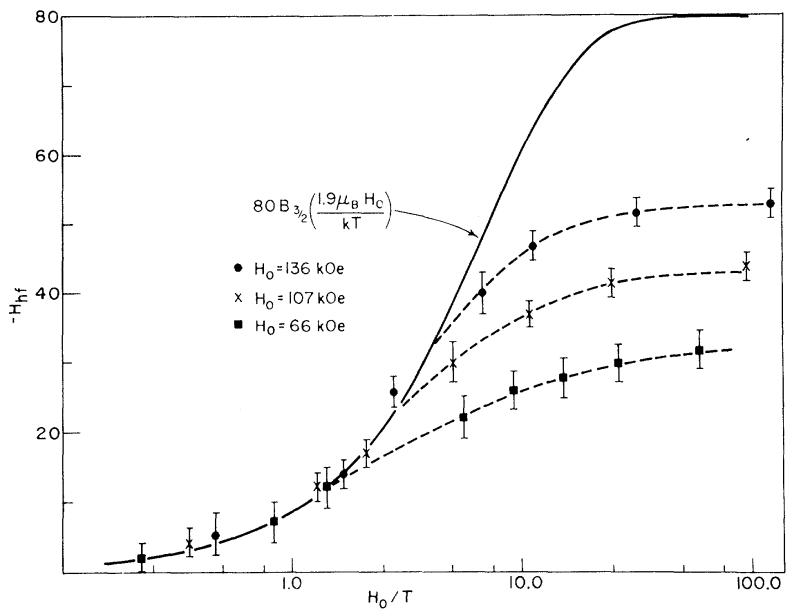

FIG. 1. $H_{\text {hf }}$ plotted as a function of $H_{0} / T$, for three values of $H_{0}$. The solid curve is a Brillouin function for $J=\frac{3}{2}$, fitted to the data at high $T$ (see text).

$\left[T<T_{\mathrm{K}}\left(H_{0}\right) ; H_{0}<H_{\mathrm{K}}(T)\right]$ where the effective moment is both temperature and magnetic field dependent; $H_{\mathrm{K}}(T)$ is the critical value of $H_{0}$, above which the bound state is destroyed. Even if a condensation to a spin-compensated bound state occurs below $T_{\mathrm{K}}$, one still expects the uncompensated spin moment $\mu^{0}$ to follow Eq. (1) for $T \gg T_{\mathrm{K}}$. The high-temperature portion of our Mössbauer data has been fitted to a Brillouin function with $J=\frac{3}{2}$ and $g=1.9$ and gives a value of $H_{\text {sat }}{ }^{0}$ corresponding to the completely unquenched moment. These values of $J$ and $g$ are taken from the magnetic susceptibility measurements of Hurd $^{15}$ in the temperature range $6^{\circ} \mathrm{K}<T<300^{\circ} \mathrm{K}$ which showed that for a large range of $\mathrm{Fe}$ concentration (15 to $220 \mathrm{ppm}$ ) the moment $\mu=g \mu_{\mathrm{B}}[J(J+1)]^{1 / 2}$ per Fe impurity in $\mathrm{Cu}$ is $(3.68 \pm 0.20) \mu_{\mathrm{B}}$. The saturation hyperfine field $\left|H_{\text {sat }}{ }^{\circ}\right|=80 \mathrm{kOe}$ is larger than the experimentally observed saturation hyperfine field $H_{\text {sat }}\left(H_{0}\right)$ for any value of applied field up to $136 \mathrm{kOe}$.

We introduce an effective moment $\mu_{\text {eff }}\left(H_{0}, T\right)$ $=f\left(H_{0}, T\right) \mu^{0}$, where $f\left(H_{0}, T\right)$ is a factor to be fitted to Mössbauer and magnetization data. The temperature- and field-dependent hyperfine field is then given by

$$
\begin{aligned}
H_{\mathrm{hf}}\left(H_{0}, T\right)=f\left(H_{0}, T\right) 80 B_{\frac{3}{2}} \\
\times\left(\frac{1.9 f\left(H_{0}, T\right) \mu_{\mathrm{B}} H_{0}}{k T}\right) \mathrm{kOe},
\end{aligned}
$$

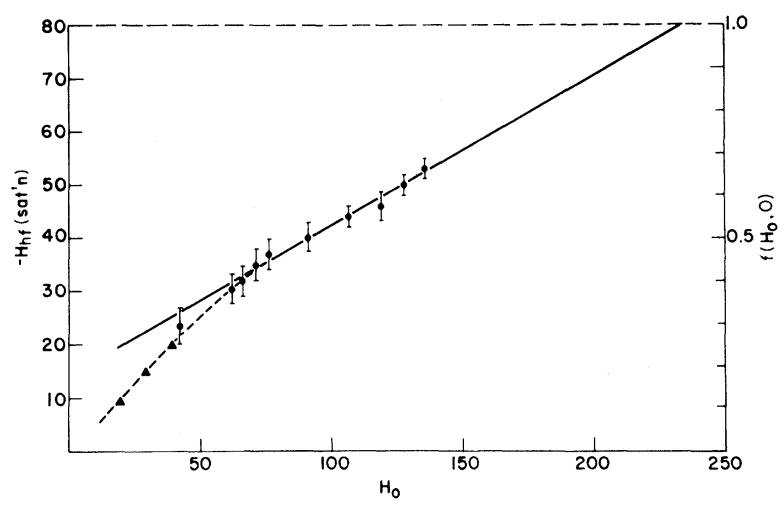

FIG. 2. The saturation hyperfine field for various values of $H_{0}$ plotted as a function of $H_{0}$. The solid curve is a linear extrapolation of the data to the theoretical full-moment hyperfine field (see text). The triangles are data points taken from Kitchens et al. (Refs. 11 and 18).

if we assume that both the saturation field and the Zeeman splitting of the electronic spin levels are proportional to the effective moment. Thus $f\left(H_{0}, 0\right)$ is equal to $H_{\text {sat }}\left(H_{0}\right) / 80 \mathrm{kOe}$ and is shown in Fig. 2. If we simply linearly extrapolate $f\left(H_{0}, 0\right)$ to 1 , we obtain an intersection at $H_{0} 235 \mathrm{kOe}$ which should be on the order of the critical field $H_{\mathrm{K}}$ necessary to destroy the bound state..$^{16,17}$

In addition to the field dependence of the effective moment we can obtain the temperature dependence of $f\left(H_{0}, T\right)$ by fitting the experimentally measured hyperfine field to Eq. (2). The $f\left(H_{0}, T\right)$ thus obtained is a monotonically increasing function of $H_{0}$ and $T$ and approaches unity whenever the values of $H_{0}$ and $T$ are such as to preclude the existence of the bound state. However, a sharp transition field or temperature is not expected, because the bound state forms gradually over a wide temperature range about $T_{\mathrm{K}} \cdot{ }^{1}$

A significant advantage of the Morssbauer technique is that it represents a microscopic measurement on an atomic scale and directly measures changes in spin polarization within atomic dimensions of the $\mathrm{Fe}$ nucleus. This is of importance because the dimension of the bound state has been suggested to be on the order of $\hbar v_{\mathrm{F}} / k T_{\mathrm{K}},{ }^{1,9}$ which for $\mathrm{Fe}$ in $\mathrm{Cu}$ is approximately $10^{-4} \mathrm{~cm}$. If the bound state consisted simply of an impurity spin surrounded by a polarized cloud of conduction electrons of opposite spin leading to a net compensation 
of the impurity moment, then the Mössbauer experiment would have measured very little field dependence of the saturation field for values of $H_{0} / T$ such that $f\left(H_{0}, T\right) g \mu_{B} H_{0} / k T \gg 1$. That is, the factor $f\left(H_{0}, T\right)$ would not appear multiplying $H_{\text {sat }}$ in Eq. (2). The field dependence of the saturation hyperfine field represents the partial breaking up the bound state and the response of the ${ }^{57} \mathrm{Fe}$ nucleus to the effective moment. Although a small intercept is obtained with a linear extrapolation of $f\left(H_{0}, 0\right)$ to $H_{0}=0$, our data do show some curvature with applied field and it seems reasonable that the $H_{0}=0$ intercept may be smaller. Mössbauer experiments in this field region are difficult because $H_{\text {sat }}\left(H_{0}\right)$ is almost equal to $H_{0}$ so that the net field at the nucleus is small and the splitting is of the order of the linewidth. The lower $T$ and $H_{0}$ measurements of Kitchens et al. ${ }^{11,18}$ indicate that $H_{\text {sat }}\left(H_{0}\right)$ extrapolates to 0 at $H_{0}=0$ (see Fig. 2). If $H_{\text {sat }}(0)=0$, then one might conclude that the bound state is a singlet. Thus the Mossbauer experiments are consistent with the formation of a bound state in the dilute $\mathrm{Fe}-\mathrm{Cu}$ alloy system resulting in a significant quenching of the impurity moment below $T_{\mathrm{K}}$. We have observed that the bound state can be strongly perturbed upon the application of a sufficiently high magnetic field. It is important to note that because the various theories predict similar behavior of the resistivity as a function of $T,{ }^{19}$ it would be useful to compare these theories as to their predictions for the magnetic behavior of dilute magnetic impurities in metals.

We are grateful to Dr. A. J. Freeman for stimulating our interest in this problem and for critical appraisal. In addition, we are pleased to thank Dr. L. W. Gruenberg, Dr. A. J. Heeger, Dr. R. M. Housley, Dr. M. A. Jensen, Dr. T. A. Kitchens, Dr. G. Knapp, Dr. J. Kondo, and Dr. W. A. Steyert for profitable discussions.
* Now at National Aeronautics and Space Administration Electronics Research Center, Cambridge, Massachusetts.

†Supported by the U. S. Air Force Office of Scientific Research.

${ }^{1}$ Y. Nagaoka, Progr. Theoret. Phys. (Kyoto) 37, 13 (1967); Phys. Rev. 138, A1112 (1965).

${ }^{2}$ P. W. Anderson, J.Appl. Phys. Suppl. 37, 1194S (1966).

${ }^{3} \mathrm{~K}$. Yosida and A. Okiji, Progr. Theoret. Phys. (Kyoto) 34,505 (1965).

${ }^{4} \mathrm{~K}$. Yosida, Phys. Rev. 147, 223 (1966); Progr. Theoret. Phys. (Kyoto) 36, 1875 (1966).

${ }^{5}$ A. Okiji, Progr. Theoret. Phys. (Kyoto) 36, 1712 (1966).

${ }^{6}$ J. Kondo, Progr. Theoret. Phys. (Kyoto) 36, 429 (1966); Phys. Rev. 154, 644 (1967).

${ }^{7}$ F. Takano and T. Ogawa, Progr. Theoret. Phys. (Kyoto) 35, 343 (1966).

${ }^{8}$ J. R. Schrieffer, J. Appl. Phys. 38, 1143 (1967).

${ }^{9}$ A. J. Heeger and M. A. Jensen, Phys. Rev. Letters 18,488 (1967).

${ }^{10}$ M. D. Daybell and W. A. Steyert, Phys。Rev. Letters $\underline{18}, 398$ (1967).

${ }^{11}$ T. A. Kitchens, W. A. Steyert, and R. D. Taylor, Phys. Rev. 138, A467 (1965).

${ }^{12}$ R. M. Housley and J. G. Dash, Rev. Mod. Phys. $\underline{36}$, 409 (1964); Phys. Letters 10, 270 (1964).

${ }^{13}$ N. A. Blum, A. J. Freeman, and L. Grodzins, Rev. Mod。Phys. 36, 406 (1964).

${ }^{14}$ A. J. Freeman and R. E. Watson, in Magnetism, edited by G. T. Rado and H. Suhl (Academic Press, Inc., New York, 1965), Vol. ILA, p. 167.

${ }^{15} \mathrm{C}$. M. Hurd, to be published; Bull. Am. Phys. Soc. 12, 348 (1967)。

${ }^{16}$ L. B. Welsh, A. J. Heeger, M. A. Jensen, and G. Gladstone in a postdeadline paper [American Physical Society, Chicago, Illinois, March, 1967 (unpublished)] reported that at very low temperature the $\mathrm{nmr}$ linewidth at the $\mathrm{Cu}$ nucleus due to the Fe impurity spin increases linearly with external field. By linearly extrapolating their data to what is expected for the full linewidth, they obtained a critical field of approximately $50 \mathrm{kOe}$.

${ }^{17} \mathrm{~B}$. Giovannini, R. Paulson, and J. R. Schrieffer, Phys. Letters 23, 517 (1966).

${ }^{18}$ The Mössbauer data of Kitchens et al. (Ref. 11) for applied fields below 62 kOe extrapolated linearly to 0 with $H_{\text {sat }}\left(H_{0}\right) \simeq 0.5 H_{0}$.

${ }^{19}$ H. Suhl, Phys. Rev. Letters 18, 743 (1967); J. Kondo, to be published. 